\title{
¿Quiénes, cómo y para qué? Los espacios de participación convocados por el Ministerio de Desarrollo Social uruguayo
}

\author{
Who, how, and for what? \\ The participation spaces convened by the Ministry \\ of Social Development of Uruguay
}

doi: http://dx.doi.org/10.32870/

espiral.v23i65.4456

Guillermo Fuentes*

Valentina Buschiazzo**

Marcelo Castillo"*

\begin{abstract}
Resumen
En este artículo se analizan los espacios de participación creados en el Ministerio de Desarrollo Social (MIDES) uruguayo entre 2005 y 2013 , considerados como instrumentos de gestión, estudiando en qué medida su funcionamiento facilita alcanzar objetivos políticos y buscando determinar si son mecanismos de innovación democrática que introducen nuevas formas de control social. Para ello, se analizan los espacios desde su posicionamiento en el cubo de la democracia (Fung, 2006). Los resultados advierten carencias en los diseños de los espacios, los recursos disponibles (humanos, materiales y de autoridad) y los criterios de convocatoria, lo cual pone en duda su potencial transformador.

Palabras clave: innovación democrática, instrumentos de gestión, participación, control social, Uruguay.
\end{abstract}

\begin{abstract}
In this article are analyzed the participation spaces created by the Ministry of Social Development (MIDES) of Uruguay between 2005 and 2013, considered management instruments, studying to what measure their function facilitates the meeting of political objectives and looking to determine if they are mechanisms of democratic innovation that introduce new manners of social control. The spaces are analyzed from their positioning in the cube of democracy (Fung, 2006). The results warned of shortcomings in the designs of the spaces, of available resources (human, material, and authoritative), and of criteria to convene, which doubts the transforming potential of the participation spaces.
\end{abstract}

Keywords: Democratic innovation, management instruments, participation, social control, Uruguay.

\footnotetext{
- Profesor-Investigador del Instituto de Ciencia Política de la Facultad de Ciencias Sociales, Universidad de la República, Uruguay. guifuen@gmail.com

- Profesora-Investigadora del Instituto de Ciencia Política de la Facultad de Ciencias Sociales, Universidad de la República, Uruguay.—valentina.buschiazzo@cienciassociales.edu.uy ••Profesor-Investigador del Instituto de Ciencia Política de la Facultad de Ciencias Sociales, Universidad de la República, Uruguay._marcelo.castillo@cienciassociales.edu.uy
} 


\section{Introducción}

La década de los ochenta del siglo pasado fue testigo a nivel global de múltiples transformaciones y cambios de paradigma en diversas arenas, consecuencia de la crisis y la pérdida de legitimidad del relativo consenso asociado a los principios del keynesianismo a nivel económico, la aspiración de universalidad de las políticas de bienestar, y una forma de organización del sector público caracterizada por sus componentes weberianos. En América Latina, dicha coyuntura devino en una serie de propuestas de reforma de orientación neoliberal, que, no obstante, tuvieron éxito dispar según cada caso (Filgueira, 2009). Con la consolidación de dichos cambios orientados a una estrategia económica promercado comenzaron a evidenciarse una serie de problemas vinculados a la falta de rendición de cuentas, responsabilidad política y crisis de representación de los partidos políticos (Cameron, et al., 2012).

Por otra parte, los mismos implicaron, en materia social, una completa redefinición del rol de las instituciones estatales en la provisión de políticas asistenciales y de bienestar a favor de una mayor intervención de organizaciones de la sociedad civil o, de forma más general, el tercer sector, o directamente del mercado (Bresser Pereira y Cunill Grau, 1998). Fue a partir de esta nueva coyuntura que se comenzaron a consolidar dos procesos de "reforma del Estado" en la región, que inevitablemente se entrelazaron: una creciente descentralización de funciones políticas, administrativas o económicas desde los niveles nacionales a instancias subnacionales, y la aparición de diversos ámbitos e instancias de participación social dentro de la administración pública para la gestión de políticas públicas (Andrenacci y Repetto, 2006).

Si bien históricamente la región ha presentado importantes desarrollos de políticas participativas, con fuerte movilización social, las mismas muchas veces estuvieron 
orientadas, por ejemplo, a organizaciones de base religiosa o movimientos insurgentes. Dichas organizaciones presentan como rasgo común el hecho de buscar deliberadamente mantener autonomía respecto a las instituciones estatales y a los canales institucionalizados de representación política, pero el cambio de época mencionado implicó un viraje en la naturaleza de los nuevos espacios y organizaciones participativas:

El tipo de participación popular que analizamos es muy distinto de las oleadas anteriores de política participativa, en la medida en que no evita los vínculos con el Estado. De hecho, en muchas ocasiones nace a partir del reconocimiento de la sociedad acerca de la incapacidad del Estado para proveer bienes públicos, lo que conduce a su vez a la aceptación de la necesidad de trabajar en conjunto para cumplir con esa tarea. En algunos casos, las instituciones para la participación directa de los ciudadanos son promovidas por el Estado (Cameron, et al., 20 I2, p. 19).

El caso uruguayo ha presentado algunas particularidades que a lo largo del siglo Xx parecieron colocarlo en un lugar excepcional dentro de la región debido a su extensa tradición en términos de democracia directa, siendo el país que registra mayor número de convocatorias a consultas populares en América Latina (Lissidini, 2012). Además de esto, la llegada al Gobierno nacional del Frente Amplio (FA) en el año 2005 destacó que, por primera vez en la historia del país, la conducción estuviera a cargo de un partido político de centro-izquierda o progresista. Este hecho, sumado a la fuerte crisis económica y social que se había producido entre los años 2002 y 2003, determinó que la agenda de gobierno presentara importantes desafíos. A nivel de políticas sociales, se produjo un refuerzo de la presencia estatal, muchas veces en materia de financiamiento y regulación, pero no tanto en términos de provisión de bienes y servicios. 
Las elecciones departamentales del año 1989 permitieron que, también por primera vez en la historia del país, un partido político de izquierda alcanzara algún cargo ejecutivo de gobierno, con la victoria del Frente Amplio en la Intendencia Municipal de Montevideo. En dicha oportunidad, parte del programa de gobierno estuvo centrado en la introducción de espacios de participación social -los presupuestos participativos- que ya se estaban convirtiendo en una moda regional a partir del éxito de la experiencia de Porto Alegre.

El impulso inicial de la propuesta fue muy fuerte. Alcanzó altos niveles de participación, que a su vez fueron respaldados por una cantidad significativa de recursos. Sin embargo, al poco tiempo de implementados, los espacios no fueron capaces de mantener esos altos niveles de involucramiento, en buena medida debido a las presiones provenientes del sistema político, las cuales derivaron en una reforma de los espacios que redujo sustantivamente la capacidad de decisión de los mismos, y, por lo tanto, minó su legitimidad (Goldfrank, 2011).

Este hecho no modificó de ninguna manera la prédica del Frente Amplio en torno a la participación social. De hecho, la propuesta programática del año 2004, cuando finalmente este partido alcanzó el Gobierno nacional, también presentaba importantes menciones a la necesidad de introducir estos espacios, fundamentalmente en el área social. En este sentido, se debían generar soluciones a los problemas de rigidez para responder a diversas demandas y a la ausencia de participación en relación con las políti-cas públicas ya consagradas (Midaglia, 2009; Midaglia y Antía, 2007), además de un cambio en la conceptualización que se hacía del sujeto de la política, ya no como un mero beneficiario que garantiza la efectividad y eficiencia a través de 
su participación, sino como un ciudadano que a través de ella ejerce sus derechos. ${ }^{1}$

Estas expectativas se fueron plasmando a lo largo de todo el espectro sectorial de políticas sociales, ya fuera a través de grandes diálogos nacionales en materia de educación y seguridad social, o introduciendo nuevos mecanismos de participación y representación de trabajadores, usuarios y, en algunos casos, empresas proveedoras al interior de los órganos de conducción de diversos organismos prestadores de políticas, como el sistema sanitario (Rodríguez Araújo, 2011).

Pero dentro de las innovaciones institucionales más importantes de ese primer periodo de gobierno, también debe incluirse la creación del Ministerio de Desarrollo Social. Entre sus cometidos fundamentales fijados por ley, se encuentra específicamente el de establecer ámbitos de coordinación y asesoramiento con la sociedad civil involucrada en los objetivos del Ministerio de Desarrollo Social (Poder Legislativo de la República Oriental del Uruguay, 2005).

A partir de este mandato legal, el nuevo ministerio ha desarrollado, desde sus diferentes unidades ejecutoras e institutos, ámbitos de participación ciudadana diversos -en cantidad y en formas de funcionamiento, recursos asociados y objetivos-, participación ciudadana que ha sido tanto directa como a través de representantes de organizaciones de la sociedad civil.

La creación del Ministerio de Desarrollo Social (MIDES) siguió una tendencia regional con un claro significado polí-

I. Por ejemplo, en dicha plataforma electoral se podían leer las siguientes sentencias: "Hasta el momento, las reformas se desarrollan sobre la base de una extensa brecha entre, por un lado, expertos y políticos, y, por otro lado, los ciudadanos. Predominan las propuestas reformistas de gabinete sin legitimidad social, [...] creemos que es necesario plantear que la transformación del Estado deberá ser parte del proceso de avance hacia una democracia auténtica, con mejoras radicales en la transparencia y permeabilidad de las decisiones, la construcción de herramientas estatales destinadas a reforzar el control horizontal, y la participación ciudadana amplia y genuina en las decisiones y el control de lo que se hace" (Frente Amplio, 2004, p. 7). 
tico: la reorganización e institucionalización de una serie de prácticas e iniciativas que se habían planteado como mecanismos de excepción para atender situaciones de vulnerabilidad social y pobreza, y que además se encontraban dispersas en el interior del aparato estatal (Midaglia, et $a l ., 2011)$. Una de las principales novedades asociadas a la nueva institución consistió en la implementación de una operativa con mayor presencia territorial que las estructuras ministeriales clásicas, y, asociado a este armado, se comenzaron a crear diversas instancias de participación de la sociedad civil organizada.

Sin embargo, la lógica de creación de estos espacios no surgió de una movilización de la sociedad demandando participar en la gestión de las políticas públicas. En el caso uruguayo, a diferencia de lo que ocurre en otros países como Brasil (Isunza y Gurza, 2010), la iniciativa surgió desde las estructuras de gobierno y se movilizó hacia abajo. Esto pudo deberse en parte a las características históricas de la relación Estado-sociedad civil en la construcción del sistema de protección social en Uruguay, que desde sus inicios ha contribuido a generar una sociedad débil y dependiente de las acciones estatales, con el correlato de un Estado fuertemente paternalista (Filgueira y Filgueira, 1994; Rossel, 2010).

En el presente artículo se propone presentar y analizar los espacios de participación creados en la órbita del Ministerio de Desarrollo Social (MIDES) en Uruguay, durante el periodo 2005-2013. Dichos espacios son considerados en función de que son instrumentos de gestión, es decir, se estudia en qué medida su funcionamiento permite o facilita la consecución de los objetivos definidos políticamente. Para ello, se parte de analizar dónde se posicionan estos ámbitos en el "cubo de la democracia" propuesto por Fung (2006).

En concreto, el objetivo general del artículo es determinar si los ámbitos de participación convocados por el MIDES pueden ser considerados mecanismos efectivos de 
innovación democrática, en el sentido de encontrarse armónicamente insertos en la estructura pública. Para ello, se caracterizarán a partir de su posicionamiento en el cubo de la democracia, para posteriormente analizar sus productos y formas de funcionamiento con base en los criterios teóricamente definidos.

El artículo se organiza de la siguiente manera: en la sección dos, se presentan las definiciones básicas a partir de las cuales se organizará el análisis -participación, interfaz socioestatal-, así como también las dimensiones que componen el cubo de la democracia; posteriormente, en la sección tres se caracterizan los ámbitos analizados a partir de las dimensiones previamente definidas; y finalmente, la sección cuatro incorpora el análisis de cada uno de los ámbitos seleccionados, para construir las respuestas a las preguntas realizadas.

\section{La participación de la sociedad civil en las políticas públicas sociales}

La participación como concepto y práctica ha ido adquiriendo múltiples acepciones a lo largo de las últimas décadas. Estas visiones diferentes en muchos casos han llegado a contraponer algunos mecanismos y procesos. Por lo tanto, es fundamental delimitar el campo de estudio de la participación como instrumento de gestión, canalizado a través de las nuevas experiencias que la literatura identifica como de innovación democrática. ${ }^{2}$

La relevancia de precisar claramente los términos utilizados responde, entre otras cosas, al hecho de que los debates

2. Dicha innovación democrática es entendida como "un proceso de creación institucional que va más allá de la promulgación de formas de participación ciudadana directa como el plebiscito, el referéndum, la iniciativa popular, y en el que se articulan modalidades continuas - no extraordinarias- de incidencia social sobre el poder público y su aparato administrativo, e incluso sobre el propio sistema político" (Isunza y Gurza, 2010, p. 2I). 
vinculados a la participación social han estado fuertemente mediados por cuestiones ideológicas a lo largo de los últimos años. Al repasar los postulados de los diferentes partidos políticos de la región -tanto de izquierda como de derecha-, así como los posicionamientos de otros actores de gran peso como los organismos financieros internacionales, puede advertirse que las cargas valorativas sobre qué implica participar, bajo qué condiciones y entre quiénes, no son materia saldada.

Brevemente, y de forma muy general, se puede establecer que las posturas provenientes de la izquierda sobre la participación pueden describirse a partir de la búsqueda de vías para la ampliación de la participación ciudadana, generando instancias de diálogo e intervención en la estructura estatal, con miras a propulsar un Estado más receptivo, responsable y abierto a las demandas sociales, al tiempo que desde la posición del ciudadano se tendería a un mayor empoderamiento que redundaría en la profundización de la democracia.

Como contrapartida, desde posiciones de derecha, la participación se ha enfocado hacia la búsqueda de mayor eficacia y eficiencia de las políticas públicas, y a mejorar la rendición de cuentas de los Gobiernos. También supone el involucramiento de actores no gubernamentales en la gestión, principalmente del sector privado. En este caso, el paradigma hegemónico de reforma administrativa promovido desde estas posiciones -la "nueva gestión pública"- ha tendido a conceptualizar al individuo como cliente o usuario, y no únicamente en su calidad de ciudadano. En definitiva, en su versión extrema, la participación más directa es la que se da en el mercado, optando por uno u otro proveedor o servicio, bajo la lógica de que "el dinero sigue al cliente".

En este mismo sentido, y como parte del proceso de reestructuración estatal, se introduce este debate al interior de la administración pública. De acuerdo con Brugué (2004), la 
modernización de la administración tiene distintos motores que se sustentan en decisiones político-ideológicas. Por un lado, desde la derecha se critica a la administración tradicional del bienestar por voluminosa, ineficiente e inoperante en la generación de política pública. No es lo que se hace, sino cómo se hace, y es por eso que propone una modificación de la administración más bien instrumental, o sea, recortar y reducir los costos del aparato administrativo. Se parte del supuesto de que el usuario de la política está siempre informado y que, por lo tanto, va a poder tomar la mejor decisión respecto a quién quiere que le ofrezca el bien o servicio y de qué manera, concibiéndolo de esta forma como cliente respecto a la toma de decisiones.

Pero mientras para la derecha el problema estaba justamente en la estructura del bienestar, para la izquierda el "Estado de bienestar" fue la víctima de la crisis (Brugué, 2004). En este caso, la modernización no se encontraba en la "mejora" vía recorte o racionalización, sino en la sustitución del instrumento. Se proponía desde esta perspectiva un cambio sustantivo que respondiera a las nuevas complejidades y demandas del orden social: cambiar el "qué" y no el "cómo" de la administración. El objetivo entonces, se desplazaba desde el interior de la administración hacia el exterior, a partir de la mejora del entorno social sobre el que intervenía.

El argumento del que parte este trabajo se apoya en las consideraciones de Isunza y Gurza sobre la convivencia de prácticas, pero con un agregado: la contraposición entre posiciones de izquierda y derecha mencionadas anteriormente adolece en sus formas de instrumentación de una falsa oposición entre democracia y gestión. Este punto se torna particularmente relevante en contextos de Gobierno de partidos de izquierda, ya que en la medida en que toda aspiración a mejorar la gestión de las políticas públicas es 
directamente asociada a una lógica tecnocrática y gerencial, las mismas quedarán bloqueadas políticamente.

¿Por qué se considera este vínculo como una falsa oposición? Básicamente por dos factores. En primer lugar, porque la gestión no es una cuestión políticamente neutra (Brugué, 2004; Narbondo, 2003), y, por lo tanto, la promoción de mejoras en las formas de hacer las cosas podrá ajustarse, o no, a los principales valores de la izquierda.

Pero además, en segundo lugar, incluso en aquellas iniciativas que no se planteen otro fin que el empoderamiento de algún sector de la ciudadanía, las mejoras en la forma de hacer las cosas dependerán en buena medida, para tener éxito, de cómo las mismas sean organizadas en términos de convocatoria, funcionamiento, que sean respaldadas tanto a nivel de recursos humanos como financieros, entre otras dimensiones. En definitiva: su éxito dependerá de cómo sean gestionados. Si se olvida este punto, existirán grandes riesgos de que se termine cayendo lisa y llanamente en un voluntarismo ingenuo.

En definitiva, lo que se está proponiendo es observar a los espacios de participación vinculados a la provisión de bienes y servicios en tanto instrumentos de gestión que pueden o no habilitar una mejora de la actividad, mientras que en aquellos casos en los que la participación aparece como el fin se pondrá el foco en cómo se gestionan los mismos.

Nada de lo dicho anteriormente pretende diluir las diferencias entre izquierda y derecha en relación a la participación, sino todo lo contrario: estas diferencias se encuentran más vigentes que nunca, pero no deben buscarse en llamados a mejor gestión o más democracia. Por el contrario, los matices y diferencias serán visualizados en otras dimensiones, como por ejemplo: la concepción del individuo que está detrás de la iniciativa, las herramientas de decisión que los participantes tendrán a disposición, el grado de vínculo de los espacios con los canales tradiciona- 
les de gobierno, y las opciones de provisión elegidas, entre otras. Por lo tanto, a pesar de la existencia de argumentos históricamente confrontados, las nuevas experiencias de innovación democrática hacen dialogar estas concepciones de forma tal que se pueden observar modelos de gestión alternativos.

Estas modalidades de incidencia social pueden ir desde altos niveles, donde los ciudadanos tienen mayor capacidad y potestad para identificar los problemas y las formas de solución de los mismos, a modalidades en las que simplemente se legitiman decisiones ya tomadas en otros ámbitos. Esto, de alguna manera, clarifica los sentidos del intercambio entre la sociedad y el Estado: cuanto más alto es el involucramiento social, mayor es la transferencia de poder desde el Estado hacia la ciudadanía.

En cambio, cuando los niveles de incidencia son más bajos, se tenderá a replicar el vínculo clásico entre burocracia y ciudadano, pero incluso en este caso, las experiencias de innovación democrática son particularmente novedosas, ya que convierten el vínculo entre Estado y sociedad en bidireccional, o en palabras de Bobbio: "el flujo del poder no puede tener más que dos direcciones: o es descendente, es decir, se mueve de arriba abajo, o ascendente, es decir, de abajo hacia arriba" (Bobbio, 2000, p. 62, citado en Rodríguez Araújo, 2011). Esta relación bidireccional es definida como “interfaz socioestatal”, otorgándole a la participación una clara inserción institucional (Isunza y Gurza, 2010).

En las nuevas instancias, se diluyen de alguna manera las fronteras entre definiciones antes contrapuestas como son las de control y participación (Isunza y Gurza, 2010). La idea de participación siempre fue depositaria de expectativas sobre el valor de la democracia, pero para medir la incidencia de la misma en la formación de políticas públicas en estas nuevas experiencias se vuelve necesario establecer algún parámetro. 
Surge así la precisión de la participación como control, algo que ya no es concebido como exclusivo de los Gobiernos en sus sistemas de pesos y contrapesos dentro de los poderes representativos, o a través de los ciudadanos con el voto en cada elección. A partir de estas nuevas experiencias institucionalizadas, el control es entendido como "la incidencia de ciudadanos sobre procesos decisorios, ya sea mediante el suministro de información, la determinación de prioridades, la implementación o como formas de evaluación y precisión" (Isunza y Gurza, 2010, p. 31).

Este concepto no simplifica la definición de participación, sino que intenta observar sus distintos efectos, que con las connotaciones morales o valorativas, y por ser relativas a un concepto abstracto, son difíciles de medir. Es decir, la definición de control no es una mera operacionalización del concepto de participación, sino que retoma un sentido específico en el que la participación es relevante, porque alude esencialmente al rol que debería jugar la misma en la gestión de políticas públicas.

Si estas instancias tienen como objetivo incidir en la política pública, deberían tener al menos tres características fundamentales (Fung y Wright, 2001).

En primer lugar, la devolución de autoridad en la toma de decisiones a unidades locales empoderadas, que sin lugar a dudas requieren de cierta reorganización del aparato estatal, es decir, la transferencia de poder administrativo y político a unidades territoriales menores, como las departamentales o locales.

En segundo lugar, la existencia de una supervisión y coordinación centralizada que implique la creación de vínculos formales de responsabilidad, distribución de recursos y comunicación que conecten estas unidades descentralizadas entre ellas y con la autoridad superior centralizada.

Y por último, un diseño centrado en el actor y no en el voluntariado, es decir, es necesario que este modelo de par- 
ticipación se encuentre formado por movimientos estructurados y organizados, para poder impactar significativamente en la configuración del Estado. Por eso mismo, esta propuesta intenta generar cambios importantes en los modos en que se organiza la administración pública, y, al mismo tiempo, institucionalizar las prácticas participativas.

A pesar de esta proliferación de espacios en la región y sus potenciales beneficios, lo cierto es que buena parte de su éxito o fracaso depende de cómo los mismos están diseñados (Hevia, 2011). Es decir: a quiénes se integra, bajo qué condiciones, con qué recursos, y cómo lo allí decidido se incorpora en la arquitectura pública de la toma de decisiones, serán aspectos centrales para definir el alcance efectivo de estos ámbitos. En síntesis, los alcances del funcionamiento de estos espacios, y por ende su capacidad de cumplir con los objetivos propuestos, van a estar condicionados por el diseño de los mismos y los formatos de regulación de su funcionamiento, en relación a las dimensiones que se detallan a continuación.

Existen diversas formas de instrumentar la participación en las políticas públicas. Una forma de describir y analizar estos mecanismos es a partir de tres dimensiones: quiénes participan, cómo los participantes intercambian información y toman decisiones, y cuál es el vínculo entre las discusiones desarrolladas en el ámbito y la acción política posterior (Fung, 2006). A partir de la distribución espacial de estas dimensiones, se puede construir "el cubo de la democracia", donde puede ubicarse cualquier mecanismo de decisión pública. Este marco es una herramienta para mirar todas las opciones, incluso en aquellos casos -alternativa predominante - en donde no hay participación ciudadana.

Dicho cubo se construye a partir de los valores que asumen los espacios analizados en tres dimensiones: quiénes participan, cómo se toman las decisiones, y el alcance de la autoridad del ámbito. De acuerdo al lugar en el espacio 
del cubo que ocupe el diseño institucional, el ámbito estará afectando en distinta medida tres importantes problemas de los Gobiernos democráticos: legitimidad, justicia y efectividad (Fung, 2006).

De esta manera, en relación a quiénes participan, las opciones pueden ir desde esquemas bien abiertos o públicos -autoselección-, hasta alternativas más restringidas donde únicamente pueden participar representantes de ciertos grupos con mayores recursos de poder. La segunda dimensión, cómo se toman las decisiones, toma en cuenta procedimientos que van desde la mera posición de espectador del actor participante, hasta una modalidad compleja de deliberación a partir del expertise técnico. Finalmente, la autoridad del espacio puede ser nula, en cuyo caso las expectativas de los participantes pasan por obtener beneficios individuales, o presentar diferentes tipos de recursos hasta llegar a la autoridad directa.

\section{Apuntes metodológicos}

La información de la que se nutre el presente artículo fue tomada en su mayor parte de la base de datos de ámbitos de participación y coordinación convocados por el MIDES. Dicha base de datos fue elaborada por el Instituto de Ciencia Política de la Facultad de Ciencias Sociales (Universidad de la República) en convenio con la Dirección Nacional de Gestión Territorial (DNGT) del MIDES.

La unidad de análisis relevada fueron los ámbitos convocados por el ministerio que presentaron determinada operación en el territorio y un sentido de permanencia en sus actividades. Es a partir de esta definición que se consideraron aquellos espacios con duración media o larga -es decir, no reuniones puntuales-convocados por el MIDES, y en los que se habilitó la participación de actores -individuales o colectivos-de la sociedad civil. Los ámbitos exclusivamente 
de participación que se convocaron desde el MIDES fueron cincuenta y cuatro en total, aunque todos tenían naturaleza diferente, debido a que allí estuvieron considerados ámbitos que funcionan en diferentes departamentos del país. ${ }^{3} \mathrm{De}$ estos cincuenta y cuatro espacios, diecisiete no devolvieron la información solicitada en los formularios, por lo que se pudieron relevar treinta y siete ámbitos de participación social.

El trabajo de campo realizado para este estudio se dividió en dos etapas: en una primera se identificaron y relevaron los espacios de participación a través de un formulario autoadministrado, aplicado a los distintos referentes de las direcciones del MIDES que tuvieron la responsabilidad, directa o indirecta (a través de alguno de sus dependientes), en la convocatoria o asistencia a espacios de participación. ${ }^{4}$ En la segunda etapa, a partir de entrevistas estructuradas se profundizó la información de forma cualitativa, por vía telefónica, con los referentes institucionales de los espacios seleccionados que previamente habían completado el formulario autoadministrado de la primera fase de relevamiento. ${ }^{5}$ En esta oportunidad, se seleccionó una muestra de espacios a partir de los siguientes criterios: unidad organizativa convocante y tipo de productos, buscando aquellos con outputs más cercanos a los cometidos fundamentales del MIDES.

\section{Los espacios de participación convocados por el MIDES: principales características}

Los espacios de participación en el Ministerio de Desarrollo Social surgieron en consonancia con el interés general

3. En Uruguay, las jurisdicciones de gobierno subnacional se denominan "departamentos".

4. Dicha fase de recolección de información se realizó entre el $1^{\circ}$ de septiembre y el 30 de diciembre de 2013.

5. Las entrevistas se realizaron entre el 23 y el 30 de abril de 2014 . 
del Gobierno por acercar a la ciudadanía al sector público y mejorar el vínculo entre ambos como forma de contribuir a la calidad y desempeño de las políticas públicas. Como se mencionó anteriormente, estos espacios se insertaron en un esquema organizativo que apuntó a alcanzar ciertos niveles de descentralización operativa, y a su vez convivieron con múltiples espacios de coordinación, tanto a nivel vertical como horizontal.

Si bien dentro del ministerio se ha discutido sobre las diferentes visiones y formas de implementar la participación, a partir de los ámbitos convocados se puede observar la falta de una estrategia unificada, con lineamientos muy laxos y poco concretos sobre qué tipo de participación promover y cuáles son las mejores herramientas para hacerlo de forma exitosa.

Dentro del organigrama ministerial, tres son los organismos que convocaron espacios de participación. Uno de ellos fue la Dirección Nacional de Gestión Territorial (DNGT), encargada de los Consejos Sociales, que son instancias presentes en todo el territorio nacional en donde la sociedad civil se reunía para discutir sobre temáticas de desarrollo social en su zona o localidad.

Por otra parte, el Instituto Nacional de la Juventud (INJU) presentó múltiples plataformas de participación, por ejemplo, el proceso de construcción del Plan Nacional de Juventudes, en donde jóvenes de todo el país dirimieron sobre sus preocupaciones en materia de salud, educación y cultura, participación, deporte, vivienda, entre otros temas importantes. Además, se encontraron bajo la órbita del INJU el programa IMPULSA (el cual es un dispositivo territorial en el que se promueve la participación juvenil a través del desarrollo de talleres y de apoyo a grupos juveniles) y el Encuentro de Arte y Juventud, que es una actividad realizada todos los años en donde asisten jóvenes de todo 
el país y donde comparten actividades artístico-culturales (danza, baile, canto, etc.).

Finalmente, el Instituto Nacional del Adulto Mayor (INMAYORES) convocó la Red de Adultos Mayores (REDAM), que fue un dispositivo territorial que concentró a las distintas asociaciones de adultos mayores para realizar actividades y discutir problemáticas que luego se retomaron en el Plan Nacional de Envejecimiento y Vejez.

Estos espacios de participación fueron creados en su totalidad entre los años 2005 y 2013, previo a la llegada al Gobierno nacional del Frente Amplio y la creación del MIDES. Entre las características generales de estos espacios vale la pena mencionar que estas instancias resultaron poco inclusivas en la participación de los beneficiarios de las políticas o prestaciones sociales, lo que sí ocurrió en instancias de evaluación.

En relación al manejo de recursos, tanto humanos como financieros, mientras que la gran mayoría de espacios (veintiocho) declaró tener recursos humanos propios, solamente nueve manejaron alguna cantidad de recursos financieros. Esta carencia de recursos propios determinó que el espacio necesitara de forma vital los aportes de los participantes para construir productos que efectivamente generaran cierto impacto.

En definitiva, los espacios de participación convocados por el MIDES difirieron en formatos, población objetivo, características y alcance territorial, pero todos pueden ser analizados a partir de las categorías del cubo de la democracia, ya que todo mecanismo que contemple la interacción con la sociedad civil, puede ser considerado en las dimensiones de ese análisis (Fung, 2006). A continuación, se presentan algunos de los espacios de participación social que fueron convocados desde el Ministerio de Desarrollo Social, identificando cuáles fueron los valores que asumieron cada uno 
de ellos en las dimensiones del cubo de la democracia ya presentadas.

\section{I. Los espacios de participación convocados por el MIDES} como instrumentos de gestión: las dimensiones del cubo de la democracia

La primera variable de análisis hace referencia al método de selección de los participantes del espacio, en este caso, quiénes formaron parte de estas instancias de acuerdo a los objetivos propuestos y a la convocatoria realizada por la dirección o instituto correspondiente. La mayoría de los espacios de participación, más allá de la unidad organizativa convocante, tuvo carácter cerrado. Esto implicó que la composición de integrantes del espacio se conociera de antemano, generando un sesgo particular en la entrada de otros actores o individuos, fundamentalmente aquellos menos organizados y con menos recursos disponibles.

El Cuadro 1 muestra las distintas dimensiones del cubo de la democracia junto a los valores adjudicados a cada espacio relevado.

Para los espacios gestionados desde el INJU, la convocatoria estuvo orientada mayoritariamente a organizaciones y personas vinculadas específicamente con la temática de juventud. Por eso, todos los espacios del INJU fueron "espacios públicos reducidos", y en la escala de Fung se posicionan en la categoría de sentar únicamente a personas $u$ organizaciones interesadas en la temática juvenil. Esto demuestra que se priorizó a personas y organizaciones con interés en las juventudes y sus temas, ya que se estima que son a priori las más dispuestas a utilizar su tiempo en participar en estos espacios: los enriquece hacia adentro, genera redes entre organizaciones y esfuerzos conjuntos para la realización de algunas actividades. 
Cuadro 1. Espacios de participación en relación a las dimensiones del cubo de la democracia

\begin{tabular}{|c|c|c|c|}
\hline $\begin{array}{l}\text { Nombre del } \\
\text { espacio }\end{array}$ & $\begin{array}{l}\text { Quiénes } \\
\text { participan }\end{array}$ & $\begin{array}{l}\text { Cómo toman } \\
\text { las decisiones }\end{array}$ & $\begin{array}{l}\text { Alcance de } \\
\text { autoridad del } \\
\text { espacio }\end{array}$ \\
\hline Consejos Sociales & $\begin{array}{l}\text { Interesados } \\
\text { profesionales }\end{array}$ & $\begin{array}{l}\text { Desarrollo de } \\
\text { preferencias }\end{array}$ & $\begin{array}{l}\text { Comunicación } \\
\text { sobre ciertas } \\
\text { demandas a } \\
\text { quienes toman las } \\
\text { decisiones }\end{array}$ \\
\hline REDAM & $\begin{array}{l}\text { Personas } \\
\text { interesadas en } \\
\text { la temática }\end{array}$ & $\begin{array}{l}\text { Desarrollo de } \\
\text { preferencias }\end{array}$ & Carácter consultivo \\
\hline $\begin{array}{l}\text { Consulta Plan } \\
\text { Nacional de } \\
\text { Juventudes }\end{array}$ & $\begin{array}{l}\text { Personas } \\
\text { interesadas en } \\
\text { la temática }\end{array}$ & $\begin{array}{l}\text { Expresión de } \\
\text { preferencias }\end{array}$ & Carácter consultivo \\
\hline IMPULSA & $\begin{array}{l}\text { Interesados } \\
\text { profesionales }\end{array}$ & $\begin{array}{l}\text { Expresión de } \\
\text { preferencias }\end{array}$ & $\begin{array}{l}\text { Beneficios } \\
\text { individuales para } \\
\text { los participantes }\end{array}$ \\
\hline $\begin{array}{l}\text { Encuentro Arte } \\
\text { y Juventud }\end{array}$ & $\begin{array}{l}\text { Interesados } \\
\text { profesionales }\end{array}$ & Espectadores & $\begin{array}{l}\text { Beneficios } \\
\text { individuales para } \\
\text { los participantes }\end{array}$ \\
\hline
\end{tabular}

Fuente: elaboración propia.

Para el caso de la DNGT, la categoría que más se ajusta en la escala propuesta por Fung (2006) es la de interesados profesionales, ya que, para este caso, se incentivó la participación de personas referentes de las instituciones y organizaciones sociales que trabajaban en el territorio. Esto no significa necesariamente que dichas personas fueran profesionales, sino que estaban interesadas en la realidad de la localidad o departamento y tenían algún conocimiento específico sobre lo que sucedía allí, y por eso se posicionaron en esta categoría.

Para los espacios de INMAYORES, el perfil de participantes es el de personas y organizaciones que estaban vinculadas a la temática de vejez y envejecimiento. Las Redes de Adultos 
Mayores (REDAM) fueron los principales ámbitos que convocaron, siendo un importante dispositivo en el territorio para la consulta sobre algunos temas, como el Plan Nacional de Envejecimiento y Vejez. En definitiva, fueron espacios que contuvieron una convocatoria relativamente selectiva de instituciones que trabajan la temática vejez y envejecimiento, ya fuera de forma directa o indirecta, con un perfil marcado de organizaciones sociales, y no de instituciones públicas.

Por lo tanto, de acuerdo a lo observado para los casos particulares correspondientes a las diferentes direcciones e institutos, puede constatarse que los mecanismos de selección de los participantes fueron cerrados, a pesar de sus diferencias, priorizando a organizaciones y, en particular, a aquellas especializadas en el tema trabajado en el espacio, salvo en los Consejos Sociales, donde la convocatoria se realizó a personas con amplio conocimiento en el territorio.

\section{2. ¿Cómo se toman las decisiones?}

Esta segunda variable hace referencia a la dinámica interna de los espacios, es decir, a cuáles son las formas que los participantes tienen para decidir y comunicarse. Asimismo, la forma en que se toman las decisiones es una de las dimensiones relevantes para observar su dinámica de trabajo y la preocupación de los participantes por comprender y manejar de forma eficiente los recursos y actividades, de acuerdo con los objetivos planteados. La deliberación entre actores participantes y la necesidad-o no-de alcanzar consensos son fundamentales en un espacio que pretende incidir en una política social concreta (Fung, 2006).

En este caso, y para la mayoría de los espacios relevados, la dinámica de participación no trascendió la sumatoria de organizaciones sociales que llegaron al ámbito con una posición determinada sobre algunos temas y una forma particular de organizarse. En términos generales, y de acuerdo 
con las entrevistas realizadas, costó generar una dinámica grupal entre los actores participantes para que el espacio no fuera solamente una sumatoria de organizaciones donde cada una planteara sus necesidades o posicionamientos.

Respecto a los posicionamientos de los distintos espacios relevados en esta variable del cubo, de acuerdo a los datos obtenidos se puede observar una concentración de las instancias en las categorías de expresión y desarrollo de preferencias como formas privilegiadas de la toma de decisiones al interior del espacio, además de algún espacio en el que las decisiones no fueron tomadas por los participantes, sino que estos concurrieron al espacio como espectadores.

A pesar de la heterogeneidad entre direcciones intraministeriales, y la multiplicidad de dinámicas en la toma de decisiones de los espacios, todos los que fueron relevados parecen dar cuenta de un proceso "minimalista", en el entendido que cualquiera de ellos estuvo lejos de aspirar a cuestiones tales como la deliberación sobre asuntos realmente significativos para el conjunto de población participante. Un proceso "minimalista" que ciertamente no genera incentivos para una dinámica de decisiones grupal y organizada, con intereses propios del espacio, y no es de los que representa a cada institución u organización que lo integra.

\section{3. Alcance de autoridad del espacio}

La última dimensión que establece este análisis es una de las más importantes, pues refiere a uno de los puntos que hacen crítico al espacio como instrumento de gestión: el alcance de autoridad que tenga el ámbito en materia de política social concreta. Como explicitaban Fung y Wright (2001), este es uno de los requisitos fundamentales para la inserción en la estructura pública de estos espacios, es decir, que a nivel central se les otorgue un grado mínimo de autoridad, en detrimento de la autoridad que pueda tener el poder central. 
La posición que ocupara cada espacio en este eje del cubo fue construida y estimada a partir de una serie de variables relevadas: la existencia o no de recursos propios, tanto humanos como financieros, junto a información obtenida de las entrevistas: el lugar que ocupó el ministerio en el espacio (con sus direcciones o institutos respectivos); el margen para incorporar nuevos temas; la capacidad de modificar aspectos del diseño y la implementación; la estabilidad y formalidad; el reconocimiento de instancias superiores de rendición de cuentas; y los tipos de incidencia del espacio respecto a la estructura central.

Para los espacios analizados, el tipo de incidencia resultó heterogéneo. En algunos, fue mayormente informativo, con niveles mínimos de influencia en las políticas, correspondiendo a la categoría beneficios individuales, ya que en este caso los espacios no tenían ningún tipo de autoridad o de lugar en la estructura de toma de decisiones, solamente pretendían generar mayor interés por la cultura o la participación, y la integración social por consiguiente. En otros, fue más vinculante, ya que permitió un feedback, al menos en forma de consulta, con las políticas programadas a nivel central y los temas que se discutían. En este caso, los espacios tuvieron un carácter altamente consultivo, de acuerdo a las categorías de Fung (2006).

Como se observa en el Cuadro 1, entre los ámbitos convocados desde el INJU, el encuentro de Arte y Juventud tuvo una incidencia informativa, con niveles mínimos de influencia en las políticas del INJU, ya que fue participativa en un sentido artístico-cultural, y no de participación ciudadana. Por este motivo, se localiza en la categoría beneficios individuales, al igual que los IMPULSA.

Para la consulta para el Plan Nacional de Juventudes (CPNJ), en cambio, fue más vinculante, ya que permitió un feedback, al menos en forma de consulta, con las políticas programadas a nivel central y los temas que se discutieron. 
El problema que presentó esta construcción del plan de acción fue la formalidad y la estabilidad. Si no se establecía un procedimiento más formalizado, pudiera que la consulta dejara de hacerse. Por esto, en términos de alcance de autoridad del ámbito, podría decirse que la CPNJ tuvo un carácter consultivo.

Por el lado de los Consejos Sociales, los mismos generaban beneficios individuales para cada organización o persona participante del ámbito con escasa intención de insertarse en la estructura pública y en la toma de decisiones del ministerio, ya que, de hecho, los entrevistados confirmaron el escaso vínculo del MIDEs con lo que en el espacio se trabajaba una vez montado.

Como contrapartida, podría decirse que la relación entre INMAYORES y las REDAM, y el tipo de incidencia del espacio sobre el instituto fueron vinculantes: los espacios tuvieron una real incidencia, en este aspecto, en la estructura de la institución convocante, principalmente por el tema del monitoreo y de la consulta para el Plan Nacional de Envejecimiento y Vejez. En este sentido, y de acuerdo a las categorías de la dimensión, el espacio tuvo un carácter altamente consultivo, ya que la relación fue de ida y vuelta en términos de saber qué temáticas trabajaban y desde qué punto de vista eran abordadas.

En síntesis, ninguno de los espacios de participación convocados por el MIDES en el periodo analizado tuvo algún tipo de capacidad resolutiva en términos de elaboración y diseño de iniciativas públicas, y se encontraron muy lejos de poder erigirse como autoridad directa. De acuerdo a las categorías del cubo, algunos espacios como las REDAM o las consultas para el Plan Nacional de Juventudes, como máximo, se constituyeron en ámbitos de carácter consultivo. Sin embargo, es bueno tener en cuenta que tales espacios tuvieron particularidades que no se encuentran en el resto de ámbitos: o bien fueron de carácter puntual para un 
macroproyecto (el Plan Nacional de Juventudes), o bien estuvieron integrados por verdaderos grupos organizados y de presión que reclamaron participación, como son los colectivos de jubilados y pensionistas a lo largo y ancho del país.

\section{Análisis: los espacios de participación del MIDES en el cubo de la democracia}

El Gráfico 1 señala a las distintas instancias de participación en el espacio tridimensional donde interactúan las tres variables del cubo de la democracia. En él, se puede observar que todos los ámbitos están ubicados en la zona más cercana al origen -o punto cero- de las tres dimensiones. En otras palabras, los espacios se posicionan en los valores más bajos de la escala.

\section{Gráfico 1. Cubo de la democracia}

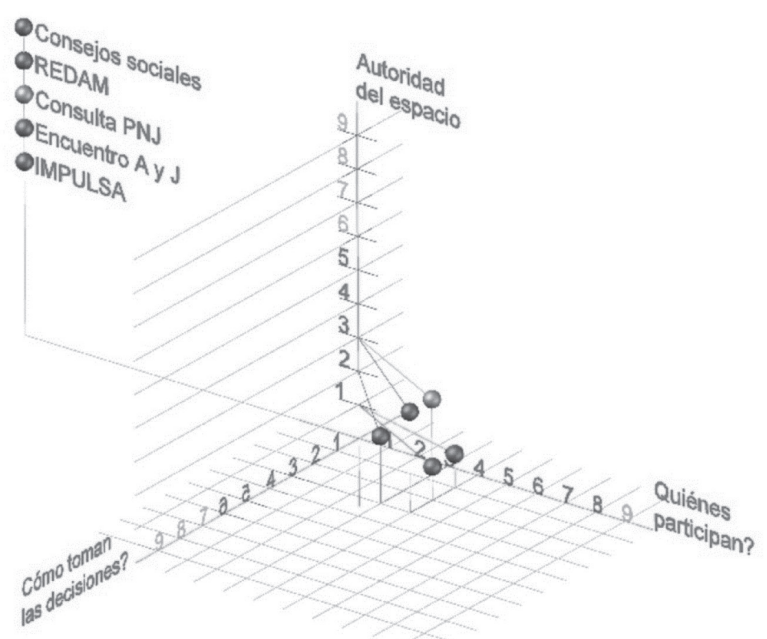

Fuente: elaboración propia con base en Fung (2006). 
De acuerdo a los datos obtenidos, las redes de adultos mayores (incluidas en la REDAM) se constituyeron como uno de los grupos de espacios mejor posicionados: parecieron insertarse de mejor forma en la estructura institucional, y ocuparon un rol claro en relación a sus áreas de acción, sin llegar con eso a generar un gran impacto en términos de acciones públicas.

Otro de los espacios que tuvo una buena posición fue la Consulta del Plan Nacional de Juventudes. Cabe destacar y recordar que, a diferencia de la REDAM, este espacio solamente se creó para un producto concreto, y no tuvo permanencia en el tiempo.

Finalmente, los espacios del Programa IMPULSA y el Encuentro de Arte y Juventud, ambos convocados por el INJU, tuvieron valores similares en el cubo de la democracia.

En este punto, vale la pena recordar que los espacios de participación, para ser considerados efectivos instrumentos de gestión, necesitarían cumplir con tres requisitos: que el espacio tuviera realmente algún tipo de autoridad real, que existiera una efectiva coordinación y rendición de cuentas respecto a la estructura central, y que el diseño del mismo se encontrara centrado en el actor y no en el voluntariado (Fung y Wright, 2001).

De lo observado se desprende que el nivel de autoridad de los espacios podría considerarse como medio-bajo o bajo, ya que no tuvieron muchas posibilidades de incidir siquiera en la modificación del diseño o implementación, y la mayoría tampoco tuvo la posibilidad de conformarse en la autoridad referida a la ejecución de políticas. Tampoco contaron con recursos financieros suficientes como para tener cierta autonomía, ni con recursos humanos con capacidades para gestionar un espacio de estas características. En el mismo sentido, tampoco pareció advertirse un vínculo institucional estable y fluido con el MIDES central. 
Los datos fueron contrapuestos para el reconocimiento de una autoridad superior y el vínculo con la estructura central. Por un lado, el vínculo con la instancia superior pareció no estar muy claro: la mayoría de los actores consideró que estos espacios funcionaron de forma aislada y que no tuvieron una instancia superior a la cual tomar como referencia. Esto afectó, sin duda, la capacidad de que los espacios generaran objetivos similares o en consonancia a los de cada unidad ejecutora, dirección o instituto del que dependieron, y, por consiguiente, afectó la inserción real de estos espacios como instancias de gestión descentralizada en el territorio.

Finalmente, otro requisito fundamental para que el espacio pudiera tener un mínimo de autoridad y de relevancia para el entramado institucional referiría a la inserción de los espacios en la estructura pública. De esta forma, el grado de institucionalización de los espacios es un dato importante, ya que es necesaria una base de formalidad y estabilidad para poder influir. De acuerdo a lo observado, los espacios que tuvieron algún tipo de formalidad a través de un marco legal o reglamentario fueron pocos, por lo tanto, desde el punto de vista institucional-formal, estos espacios no estuvieron consolidados, no ostentaron un lugar en la estructura pública formal.

Respecto a la convocatoria y conformación de estos espacios, es decir, quiénes participaron, se puede decir que, de acuerdo a la información relevada y a las categorías teóricas manejadas en este trabajo, los mismos fueron poco inclusivos, ya que la convocatoria pareció concentrada en "interesados profesionales" y en "personas vinculadas al tema del ámbito", esto en términos de Fung. Por otra parte, la convocatoria en casi todos los casos fue cerrada, y la invitación dependió exclusivamente de la voluntad de la institución convocante, lo que fue en contra del potencial democratizador de estos espacios, de alguna manera. 
Además, los actores convocados fueron instituciones de la sociedad civil organizada, lo que implicó niveles previos de socialización, lo que justamente es una de las carencias más importantes de la población con la que trabajan estos ámbitos. El hecho que los beneficiarios rara vez participaran del diseño o de la evaluación de las intervenciones también reforzó la orientación de los espacios para la gestión, en detrimento del objetivo socializante e integrador.

Como ya se mencionó, los espacios relevados tuvieron grandes dificultades para posicionarse como ámbitos en donde se decidiera alguna cuestión fundamental en materia de políticas del MIDES en el territorio, o que pudiera generar alguna modificación en el diseño o implementación de sus políticas, ya que la mayoría a priori no fue pensada para eso. Fueron esencialmente espacios de integración de organizaciones en el departamento o localidad, en donde los objetivos principales fueron la consulta, la expresión de preferencias, y, en algún caso, el desarrollo de las mismas por parte de los participantes. Por ende, en relación a la incidencia del ámbito, de acuerdo a las categorías teóricas presentadas en este informe, la misma fue en casi todos de carácter meramente informativo.

Retomando las dimensiones del cubo de la democracia, se observa claramente cómo los ámbitos no presentaron grandes diferencias entre sí, independientemente de la dirección o instituto que convocó, al tiempo que se estructuraron para generar dos tipos de productos: beneficios individuales, por un lado, en los que daría la sensación que se estuvo bastante lejos de llegar a ejecutar acciones directas y sustantivas, y la consulta sobre algunos temas. Por ende, la autoridad efectiva de los mismos fue baja. 


\section{Consideraciones finales}

Ampliar la voz de diferentes colectivos sociales, fundamentalmente aquellos más marginados o excluidos, parece ser una tarea imprescindible para fortalecer las capacidades democráticas y generar mayores mecanismos de inclusión social en los países de América Latina.

La voz puede fortalecer a las instituciones formales de la democracia representativa al incluir a los ciudadanos subrepresentados, al enseñar a los ciudadanos a representarse a sí mismos, a lograr que los representantes electos sean más sensibles a la ciudadanía y rindan cuentas (Cameron y Sharpe, 2012, pp. 342-343).

Pero reconocer esta importancia política y académica no quiere decir que existan consensos en relación a los impactos de las nuevas modalidades de participación social -innovaciones democráticas- sobre la democracia y la gestión pública. Sobre todo en este segundo punto es que el presente trabajo ha pretendido aportar algunos elementos presentes en el caso uruguayo, y, en particular, en la arena de asistencia social.

De los elementos presentados a lo largo del artículo, se desprende que tanto el diseño institucional, como los recursos y actividades llevadas adelante por los espacios de participación convocados desde el MIDES, presentan importantes déficits si se les conceptualiza como claves de instrumentos de gestión. De hecho, difícilmente pueden ser entendidos como mecanismos de innovación democrática para la gestión de políticas sociales en la medida en que ni concretaron amplios niveles de autoridad y capacidad de decisión sobre asuntos constitutivos del accionar ministerial, ni tampoco terminaron de consolidarse como espacios que oficiaran como legitimadores de decisiones tomadas en otros espacios. 
Este hecho terminó dejando como opción una tendencia a que estos ámbitos se consolidaran como espacios autoreferidos que en última instancia podrían terminar agotándose en sí mismos con los problemas asociados en términos de mantener la legitimidad del ámbito y el compromiso de los participantes.

Esta situación podría explicarse -aunque no solamentepor algunos problemas vinculados al diseño de los mismos. En este sentido, la posición que ocupan estos espacios en el cubo de la democracia es de gran utilidad, ya que de allí se pueden extraer algunas conclusiones sobre los cambios que podrían realizarse en pos de un futuro rediseño. Así, el gran punto parece ser la escasa autoridad que tuvieron los ámbitos para lograr impactar efectivamente en una política o población a través de sus decisiones. Colaboran poco en este sentido dos factores: por un lado, no aparecer asociados íntimamente a instancias u organismos ejecutores, ni tampoco manejar recursos propios que les permitieran asumir esta tarea; pero, por otra parte, no tener autoridad -y por ende no ser expresión de algún tipo de devolución de poder a instancias subnacionales o locales- por ser mecanismos de gestión pública que se insertaron en un entramado institucional fuertemente centralizado. En definitiva, la idea es que difícilmente se podrá construir autoridad en espacios de este tipo si dicho proceso no aparece de la mano de una descentralización efectiva, en este caso, del organismo convocante y algunos de los participantes más importantes.

Un segundo fenómeno, a partir del caso analizado, que debería encontrarse presente para garantizar un mejor funcionamiento de los espacios de participación es un adecuado vínculo entre los mismos y algunas instancias de coordinación, tanto a nivel horizontal -territorial- como vertical -entre niveles de gobierno-, ya sean estas a nivel intersectorial o en el diálogo exclusivo con un ministerio. La razón es sencilla: este vínculo podría oficiar como sustituto 
de la existencia de recursos disponibles para la ejecución, ya que, entre otras cosas, garantizaría una adecuada inserción en el entramado institucional existente, evitando el aislamiento advertido.

Entonces, si lo dicho hasta aquí es aceptado, el mayor valor de estos espacios podría encontrarse en cierto tipo de componente simbólico para la formación de un espacio público donde la población pueda reunirse y discutir, pero con escaso impacto a nivel de las políticas del MIDES. Sin embargo, también este escenario presenta dificultades para concretarse efectivamente, ya que el diseño y gestión de estos espacios está orientado a la participación de actores sociales organizados, quedando por fuera de prácticamente toda instancia los beneficiarios de los planes y programas del MIDES, en particular, pero, en general, también los ciudadanos considerados individualmente. Es decir: ni tan orientado a la gestión, ni tan abierto a la ciudadanía.

En definitiva, la multiplicidad de realidades en los ámbitos existentes hace que todavía se esté muy lejos de consolidar a estos espacios como mecanismos de innovación democrática orientados a la mejora de la gestión pública y de empoderamiento ciudadano. La inserción institucional actual de estos espacios, así como las configuraciones de funcionamiento existentes, dificultan en demasía la acumulación y el avance hacia alguno de estos objetivos.

Como puntos a tener en cuenta a la hora de pensar un rediseño de los mismos, podrían mencionarse fundamentalmente dos: 1) insertar a estos ámbitos en un esquema efectivo de coordinación, tanto vertical como horizontal, para otorgarle mayor legitimidad y alcance a lo que allí se genere; y 2) recordar que la "población MIDES" requiere de una serie de acciones afirmativas que compensen ciertos déficits de partida, y que, por lo tanto, es fundamental integrar a estas personas en espacios que puedan ser apropiados en el sentido de compensar los déficits o desigualdades pre- 
existentes, y, sobre todo, donde efectivamente lo acordado se vea materializado en una acción pública.

Sin negar el valor potencial de estos espacios en el funcionamiento cotidiano de un ministerio como el de Desarrollo Social, la información presentada parece apoyar la idea de que todavía es necesario continuar perfeccionando la herramienta en pos de lograr que la misma contribuya a alcanzar objetivos públicos de gran importancia.

Fecha de recepción 31 de octubre de 2014

Fecha de aceptación 22 de junio de 2015

Andrenacci, L., y Repetto, F. (2006). "Ciudadanía y capaciBibliografía dad estatal: dilemas presentes en la reconstrucción de la política social argentina", en L. Andrenacci (comp.), Problemas de política social en la Argentina contemporánea (pp. 289-319). Buenos Aires: UNGS-Editorial Prometeo. Bresser Pereira, L. C., y Cunill, N. (1998). "Entre el Estado y el mercado: lo público no estatal”, en L. C. Bresser, y N. Cunill (eds.), Lo público no estatal en la reforma del Estado (pp. 25-58). Buenos Aires: CLAD, Paidós.

Brugué, Q. (2004). “Modernizar la administración desde la izquierda: burocracia, nueva gestión pública y administración deliberativa". Reforma y Democracia, (29), I- 16. Recuperado de: http://siare.clad.org/revistas/0048900. pdf

Cameron, M., Hershberg, E., y Sharpe, K. (20I2). Nuevas instituciones de democracia participativa en América Latina: la voz y sus consecuencias. México: FLACSO.

-, y Sharpe, K. (20I2). "La voz institucionalizada en las democracias de América Latina", en M. Cameron, E. Hershberg, y K. Sharpe (eds.), Nuevas instituciones de democracia participativa en América Latina: la voz y sus consecuencias (PP. 337-36I). México: FLACSO. 
Bibliografía
Filgueira, C. H., y Filgueira, F. (1994). El largo adiós al país modelo: políticas sociales y pobreza en Uruguay. Montevideo: Editorial Arca.

Filgueira, F. (2009). El desarrollo maniatado en América Latina: Estados superficiales y desigualdades profundas. Buenos Aires: Consejo Latinoamericano de Ciencias SocialesCLACSO.

Frente Amplio (2004). "Grandes lineamientos programáticos para el Gobierno 2005-2010. Porque entre todos otro Uruguay es posible". IV Congreso Extraordinario. Recuperado de: www.frenteamplio.org.uy/index. php? $Q=$ download\&ID $=1040$

Fung, A. (2006). "Varieties of participation in complex Governance”. Public Administration Review, 66(issue supplements I), 66-75. Recuperado de: http://www. archonfung.net/papers/FungVarietiesPAR.pdf

—, y Wright, E. (200I). Deepening Democracy Institutional Innovations in Empowered Participatory Governance. Nueva York: Verso.

Hevia, F. (20I I). "Participación ciudadana institucionalizada y organizaciones civiles en Brasil: articulaciones horizontales y verticales en la política de asistencia social". Revista de Estudios Sociales, (30), 95- 108.

Isunza, E., y Gurza, A. (2010). La innovación democrática en América Latina. Tramas y nudos de la representación, la participación y el control social. México: Publicaciones de la Casa Chata.

Lissidini, A. (2012). "Democracia directa en Uruguay y en Venezuela: nuevas voces, antiguos procesos", en $\mathbf{M}$. Cameron, E. Hershberg, y K. Sharpe (eds.), Nuevas instituciones de democracia participativa en América Latina: la voz y sus consecuencias (Pp. 235-27I). México: FLACSO.

Midaglia, C. (2009). "Entre la tradición, la modernización ingenua y los intentos de refundar la casa: la reforma social en el Uruguay de las últimas tres décadas", en C. 
Barba (ed.), Retos para la integración social de los pobres Bibliografía en América Latinam (pp. 85- I07). Buenos Aires: CLACSO. -, y Antía, F. (2007). "La izquierda en el Gobierno: ¿cambio o continuidad en las políticas de bienestar social?". Revista Uruguaya de Ciencia Política, (16), I3I157.

O'Donnell, G. (2004). “Notas sobre la democracia en América Latina”, en PNUD (coord.), La democracia en América Latina. Hacia una democracia de ciudadanas y ciudadanos. El debate conceptual sobre la democracia (pp. I I-82). Buenos Aires: PNUD.

Peruzzotti, E., y Selee, A. (2009). "Participatory innovation and representative democracy in Latin America", en A. Selee, y E. Peruzzotti (eds.), Participatory innovation and representative democracy in Latin America (pp. I- 16). Estados Unidos: Johns Hopkins University Press.

Poder Legislativo de la República Oriental del Uruguay (2005). Ley 17.866. Creación del Ministerio de Desarrollo Social. Recuperado de: http://www.parlamento.gub.uy/ leyes/AccesoTextoLey.asp? Ley= I 7866\&Anchor=

Rodríguez Araújo, M. (20I I). Democratización de la gestión pública en el Gobierno del Frente Amplio. El caso de la participación social en la reforma del sector salud (monografía final de la Licenciatura de Ciencia Política). Facultad de Ciencias Sociales, Universidad de la República: Montevideo.

Rossel, C. (2010). "Tercer sector y prestación de servicios sociales públicos: la 'caja negra' de la participación ciudadana en la gestión pública y su impacto en los regímenes de bienestar corporativos". Reforma y Democracia, (47). Recuperado de: http://www.redalyc.org/articulo. oa?id=357533679007 